\title{
ANTICONCEPCIONAIS DE EMERGÊNCIA - POR QUE NÃO USAR?
}

\author{
EMERGENCY CONTRACEPTIVES: WHY NOT USE THEM?
}

Antonio Alberto Nogueira' ${ }^{1}$; Francisco José Candido dos Reis ${ }^{1} \&$ Omero Benedicto Poli Neto ${ }^{2}$

\begin{abstract}
${ }^{1}$ Docentes; ${ }^{2}$ Médico Residente. Departamento de Ginecologia e Obstetrícia - Faculdade de Medicina de Ribeirão Preto-USP. CoRRESPondÊncia: Departamento de Ginecologia e Obstetrícia - Faculdade de Medicina de Ribeirão Preto - USP. Campus Universitário CEP: 14048-900 - Ribeirão Preto, São Paulo.
\end{abstract}

NOGUEIRA AA; REIS FJC \& POLI NETO OB. Anticoncepcionais de emergência - por que não usar? Medicina, Ribeirão Preto, 33: 60-63 jan./mar. 2000.

RESUMO: Artigo de revisão sobre métodos anticoncepcionais de emergência, abordando seu mecanismo de ação, eficácia e indicações, ilustrado com questionário informal, realizado com estudantes do primeiro ano do Curso de Ciências Médicas da FMRP-USP. O inquérito mostrou ser o método pouco conhecido e os que o conheciam apresentaram dúvidas sobre seu mecanismo de ação e efeitos adversos.

UNITERMOS: Anticoncepcionais Pós-Coito. Anticoncepcionais. Emergências.

\section{INTRODUÇÃO}

Os anticoncepcionais de emergência, também chamados pós-coitais ou do dia seguinte, são métodos alternativos de anticoncepção para serem usados em situações especiais, principalmente nos casos de:

1. relação sexual desprotegida, não planejada;

2. uso inadequado de métodos anticoncepcionais, como, por exemplo esquecimento de duas ou mais pílulas;

3. falha anticoncepcional presumida, como, por exemplo, "acidentes" com o uso do preservativo (rotura ou retenção na vagina) ou com o diafragma;

4. violência sexual.

Vários são os métodos utilizados para esse fim, entre eles os relatados a seguir.

\subsection{Anticoncepcionais Orais}

Os regimes anticoncepcionais orais mais utilizados são os combinados, que contêm uma associação de estrogênios e progestogênios, e/ou os de progestogênios puros.
O combinado, conhecido também como regime de "Yuzpe"(1), consiste na ingestão de duas doses de $100 \mathrm{mcg}$ de etinil estradiol e $500 \mathrm{mcg}$ de levonorgestrel em duas tomadas, com intervalo de 12 horas, sendo a primeira o mais próximo possível do relacionamento sexual desprotegido, e, no máximo, 72 horas após. O regime de progestogênio puro consiste na ingestão de duas doses de $750 \mathrm{mcg}$ de levonorgestrel com intervalo de 12 horas entre elas, também o mais precoce possível e, no máximo, 72 horas após a relação sexual desprotegida, já que sua eficácia pode diminuir, quando postergada ${ }^{(2,3)}$.

\subsubsection{Mecanismo de ação}

Dependendo da fase do ciclo menstrual em que é usado, pode interferir com a ovulação - supressão ou atraso; com a nidação - altera a resposta endometrial, ou - com a fecundação - pode alterar a função do corpo lúteo e a motilidade tubária. Apesar de sua eficácia constatada, uma vez iniciado o processo de nidação, tais anticoncepcionais perdem a efetividade, e, no caso de falha, não há evidências de efeitos nocivos para o embrião ou piora no prognóstico da gestação ${ }^{(4,5,6)}$. 


\begin{tabular}{|c|c|c|c|c|c|}
\hline \multicolumn{6}{|c|}{ Formulação e dose das pílulas anticoncepcionais de emergência } \\
\hline \multicolumn{2}{|r|}{ Formulação } & $\begin{array}{c}\text { Nome } \\
\text { Comercial }\end{array}$ & $\begin{array}{l}\text { № de comp. } \\
\text { por dose }\end{array}$ & $\begin{array}{l}\text { № de } \\
\text { doses }\end{array}$ & $\begin{array}{c}\text { Período de } \\
\text { administração }\end{array}$ \\
\hline Combinados & $\begin{array}{l}\text { Levonorgestrel } 250 \mathrm{mcg}+ \\
\text { E. Estradiol } 50 \mathrm{mcg} \\
\text { Levonorgestrel } 150 \mathrm{mcg} \\
\text { E. Estradiol } 30 \mathrm{mcg}\end{array}$ & $\begin{array}{l}\text { Neovlar } \AA \\
\text { Evanor }{ }^{\circledR} \\
\text { Microvlar }{ }^{\circledR} \\
\text { Nordete } \AA\end{array}$ & $\begin{array}{l}2 \\
2 \\
4 \\
4\end{array}$ & $\begin{array}{l}2 \\
2 \\
2 \\
2\end{array}$ & \multirow[t]{2}{*}{$\begin{array}{l}\text { Primeira dose até } 72 \\
\text { horas após coito des- } \\
\text { protegido, segunda } \\
\text { dose } 12 \text { horas após }\end{array}$} \\
\hline $\begin{array}{c}\text { Progestogênio } \\
\text { Puro }\end{array}$ & Levonorgestrel $750 \mathrm{mcg}$ & Postinor $2 \AA$ & 1 & 2 & \\
\hline
\end{tabular}

\subsubsection{Efeitos colaterais}

O principal efeito colateral dos anticoncepcionais combinados são as náuseas e vômitos. Aproximadamente metade das usuárias apresentam náuseas e $22 \%$, vômitos. Já os regimes com progestogênios puros apresentam uma incidência menor, $16 \%$ de náuseas e 3\% de vômitos. Quando os vômitos ocorrem até duas horas após a ingestão, a dose deve ser repetida. Outros efeitos secundários, associados com ambos os regimes, em porcentagens menores, incluem: cansaço, aumento da sensibilidade das mamas e cefaléia, geralmente não durando mais de 24 horas $^{(7)}$.

A maioria das pacientes tratadas sangra 14 a 21 dias após o tratamento.

\subsection{Mifepristone}

O Mifepristone (anti-progestogênio), utilizado em dose única de 10 a 600 mcg, tem se mostrado tão efetivo quanto o regime de Yuzpe no tratamento anticoncepcional de emergência e com menos efeitos colaterais, como as náuseas e vômitos. Apresentam, contudo, uma maior incidência de atraso no sangramento menstrual, pós-tratamento, talvez, devido ao seu efeito antiprogestogênico, podendo levar a um atraso na ovulação, quando utilizado na primeira fase do ciclo menstrual $^{(8)}$ (ainda não disponível em nosso meio).

\subsection{Dispositivo Intra-Uterino}

O DIU pode ser uma opção anticoncepcional de emergência para algumas mulheres, principalmente para aquelas que desejam continuar a usá-lo como método anticoncepcional de longa duração. Trata-se de um método efetivo e seguro. Em um estudo com 515 pacientes, cujo DIU foi inserido até cinco dias após a relação desprotegida, ocorreram dois casos de falha do método, que resultaram em gestações, a taxa de remoção foi de $14,9 \%$ nas pacientes nulíparas e $3,5 \%$ nas multíparas, não houve falha no procedimento de inserção ou infecção pélvica associada ao procedimento $^{\left({ }^{(9)}\right.}$.

As mulheres que fazem opção por tal método devem ser submetidas aos mesmos critérios de uso a que são submetidas as usuárias regulares desse método. Podem sofrer os mesmos efeitos colaterais, que são os principais responsáveis pela descontinuidade do método, ou seja, aumento do sangramento menstrual e da dismenorréia ${ }^{(10)}$.

\section{RESULTADOS DE UM INQUÉRITO}

Apesar de sua segurança e alta eficácia, esses métodos ainda são pouco usados, pelo desconhecimento de sua existência, de sua forma de uso, de seus mecanismos de ação, pela falta de formulações específicas aprovadas para esse uso ou pelas informações distorcidas a respeito de seus mecanismos de ação e de sua legalidade.

Em inquérito que procurou avaliar o nível de conhecimento sobre anticoncepção de emergência, entre 91 estudantes do $1^{\circ}$ ano da FMRP-USP, no ano de 1999, com idade média de 19,1 anos (31 do sexo feminino e 60 do sexo masculino) oito não conheciam o método, e, entre os 83 que o conheciam, encontramos os dados mostrados nas Tabelas de I a IV.

Entre os que não usariam, a maioria, em ambos os sexos, acreditava ser o método abortivo e tinha dúvidas sobre os seus efeitos colaterais.

Além da preocupação com os efeitos colaterais, que são poucos e fugazes, principalmente para a maioria das usuárias de anticoncepcionais de emergência, jovens e saudáveis, uma grande preocupação 


\begin{tabular}{|c|c|c|c|}
\hline & $\begin{array}{l}\text { Sexo Mas- } \\
\text { culino }\end{array}$ & $\begin{array}{l}\text { Sexo femi- } \\
\text { nino }\end{array}$ & Total \\
\hline Não sabiam & $7(12,7 \%)$ & $5(17,9 \%)$ & 12 \\
\hline Sabiam parcialmente & $48(87,3)$ & $23(82,1 \%)$ & 71 \\
\hline Total & 55 & 28 & 83 \\
\hline
\end{tabular}

Tabela II - Conhecimento sobre as indicações de uso dos anticoncepcionais de emergência

\begin{tabular}{|lccc|}
\hline & $\begin{array}{c}\text { Sexo } \\
\text { Masculino }\end{array}$ & $\begin{array}{c}\text { Sexo } \\
\text { Feminino }\end{array}$ & Total \\
\hline Não conheciam & $13(46,4 \%)$ & $18(32,7 \%)$ & 31 \\
Conheciam & $12(42,9 \%)$ & $25(45,5 \%)$ & 37 \\
parcialmente & & & \\
Conheciam bem & $3(10,7 \%)$ & $12(21,8 \%)$ & 15 \\
Total & 28 & 55 & 83 \\
\hline
\end{tabular}

dos usuários e até dos profissionais médicos é a crença de que os anticoncepcionais de emergência seriam microabortivos. Como já vimos, anteriormente, o mecanismo de ação desses anticoncepcionais é, sem dúvida, múltiplo, incluindo também alterações endometriais que poderiam inibir a implantação. Essas alterações endometriais não são exclusivas desse método e, sim, da maioria dos métodos hormonais e até naturais como em algumas fases da amamentação, com ciclos ovulatórios mas com endométrio alterado por insuficiência lútea e, portanto, inadequado para a

\begin{tabular}{|c|c|c|c|}
\hline & $\begin{array}{c}\text { Sexo } \\
\text { Masculino }\end{array}$ & $\begin{array}{c}\text { Sexo } \\
\text { Feminino }\end{array}$ & Total \\
\hline Não conheciam & $19(34,5 \%)$ & $09(32,1 \%)$ & 28 \\
\hline $\begin{array}{l}\text { Conheciam um } \\
\text { método }\end{array}$ & $21(38,2 \%)$ & $12(42,9 \%)$ & 33 \\
\hline $\begin{array}{l}\text { Conheciam mais } \\
\text { de um método }\end{array}$ & $15(27,3 \%)$ & $7(25,0 \%)$ & 22 \\
\hline Total & 55 & 28 & 83 \\
\hline
\end{tabular}

\begin{tabular}{|c|c|c|c|}
\hline & $\begin{array}{c}\text { Sexo } \\
\text { Masculino }\end{array}$ & $\begin{array}{c}\text { Sexo } \\
\text { Feminino }\end{array}$ & Total \\
\hline Não responderam & $08(14,5 \%)$ & 03 (10,7\%) & 11 \\
\hline Sim & $20(36,4 \%)$ & $13(46,4 \%)$ & 33 \\
\hline Não & $27(49,1 \%)$ & $12(42,9 \%)$ & 39 \\
\hline Total & 55 & 28 & 83 \\
\hline
\end{tabular}

nidação. A própria gravidez humana tem uma eficácia pequena e, mesmo em casais normais, quando há fecundação, mais de $60 \%$ dos blastocistos não se implantam e nem por isto são caracterizados como aborto. Não se justifica, portanto, essa preocupação com os anticoncepcionais de emergência, e, sim, com a orientação dos usuários e sua disponibilidade para prevenção de uma gravidez indesejada. Graves problemas de saúde pública podem resultar das interrupções destas gestações, muitas vezes ilegais e com alto risco para a mãe ${ }^{(11,12,13)}$.

NOGUEIRA AA; REIS FJC \& POLI NETO OB. Emergency contraceptives: why not use them? Medicina, Ribeirão Preto, 33: 60-63, jan./march 2000.

ABSTRACT: This is a review article concerning emergency contraceptives, with a description of their mechanism of action, efficacy and indications, ilustrated with na informal questionnaire applied to first-year medical studients at FMRP-USP. The questionnaire showed that the method is not well known and those students who knew them had doubt about their mechanisms of action and adverse effects.

UNITERMS: Contraceptives, Postcoital. Contraceptive Agents. Emergencies. 


\section{REFERÊNCIAS BIBLIOGRÁFICAS}

1 - YUZPE A; SMITH RP \& RODEMARKER AW. A multicenter clinical investigation employing ethinyl estradiol combined with dl-norgestrel as a postcoital contraceptive agent. Fertil Steril 37: 508-513, 1982.

2 - TASK FORCE ON POST-OVULATORY METHODS OF FERTILITY REGULATION. Randomized controlled trial of levonorgestrel versus the Yuzpe regimen of combined oral contraceptives for emergency contraception. Lancet 352: 428-33, 1998.

3 - PIAGGIO G; VON HERTZEN H; GRIMES DA \& VAN LOOK PFA. Tim ing of emergency contraception with levonorgestrel or the Yuzpe regimem. Lancet 353: 721, 1999.

4 - LING WY; ROBICHAUD A; ZAID I; WRIXON W \& MAC LOOD SC. Mode of action of dl-norgestrel and ethinyl estradiol combination in postcoital contraception. Fertil Steril 32: 297-301, 1979.

5 - BRACKEN MB. Oral contracption and congenital malformations in offspring: a review and meta-analysis of the prospectives studies. Obstet Gynecol 76: 552-557, 1990.

6 - WEBB A \& TABERNER D. Clotting factors after emergency contraception. Adv Contracept 9: 75-82, 1993.

7 - HO PC \& KWAN MSW. A prospective randomized comparison of levonorgestrel with the Yuzpe regimen in postcoital contraception. Hum Reprod 8: 389-392 , 1993.
8 - TASK FORCE ON POST-OVULATORY METHODS OF FERTILITY REGULATION. Comparison of three single doses of mifepristone as emergency contraception: a randomised trial. Lancet 353: 679-702, 1999.

9 - LIYING Z \& BILIAN X. Preliminary analysis of a multicenter clinical trial using Multiload Cu 375SL for emergency contraception. Adv Contracept 14:161-70, 1998.

10 - FASOLI M; PARAZINI F; CECCHEPTI G \& LA VECCHIA C. Postcoital contraception: a overview of published studies. Contraception 39: 459-468, 1989.

11 - GLASIER A. Emergency contraception: time for de-regulation? Br J Obstet Gynecol 100: 611-612, 1993.

12 - TRUSSELL J; ELLETSON C \& RODRIGUES G. The effectiveness of the Yuzpe regimen of emergency contraception. Fam Plann Perspect 28: 58-64, 1996.

13 - BAGSHAW S; EDWARD D \& TUCKER AK. Ethinyl Oestradiol and D-Norgestrel is a effective emergency postcoital contraceptive: AUST N Z J Obstet Gynecol 28: 137-140, 1998.

Recebido para publicação em 20/10/1999

Aprovado para publicação em 03/03/2000 